\title{
Conceptual Design for a Coaxial Ratcheting Continuously Variable Transmission
}

\author{
Kuen-Bao Sheu \\ Shih-Yuan Lai \\ Department of Vehicle Engineering \\ National Formosa University \\ Huwei, Yuenlin 63208, Taiwan, ROC
}

\begin{abstract}
A ratcheting continuously variable transmission (CVT) comprises a ratcheting drive unit, an output drive unit and a speed control unit. This study uses creative design methodology to design a ratcheting drive unit for a coaxial ratcheting CVT. Current designs for a ratcheting CVT are categorized in terms of topological characteristics to determine the design requirements and design constraints for new designs. Number synthesis is used to determine all of the feasible generalized chains for a ratcheting drive unit, including a three-bar to five-bar mechanism with one degree of freedom. Specialized chains are then specified, subject to certain design requirements and constraints. Each specialized chain is then particularized and a schematic diagram of each chain for the ratcheting drive unit is presented. Ten new designs of coaxial ratcheting CVT are then generated using the output drive unit and speed control unit. A kinematic analysis of an example design uses the vector loop method to verify the feasibility of the design.
\end{abstract}

Keywords: Transmission; Ratcheting CVT; CVT; IVT; Creative design methodology

\section{Introduction}

A continuously variable transmission (CVT) changes smoothly through a continuous range of gear ratios. This contrasts with other mechanical transmissions generally use a fixed number of gear ratios. One type of CVT is an infinitely variable transmission (IVT), for which the range of ratios of output shaft speed to input shaft speed includes a zero ratio. A CVT allows a mechanical device to operate within a particular speed or torque range and CVT's find applications in vehicles, motorcycles and supply devices for automated machinery. Mechanical CVTs include hydrostatic CVT's, traction CVT's, belt CVT's and ratcheting CVT's.

A ratcheting CVT is a transmission that comprises a ratcheting drive unit, an output drive unit and a speed control unit. The ratcheting drive unit uses a set of elements as a pulse generator to transform a rotational movement into an oscillating movement. The output drive unit often uses one-way clutches or ratchets to convert the oscillatory motion into the one-way rotation of the output system. The speed control unit allows smooth and continuous changes within a certain range by changing the linkage geometry within the ratcheting drive unit.

The mechanisms in the ratcheting drive unit of a ratcheting CVT include a linkage mechanism and a cam mechanism. A linkage-type ratcheting CVTs uses several reciprocating four-bar linkages to oscillate the ratcheting clutches (Yates, W. A., 2008; Matsumoto, S., Inoue, N., \& Tsukada, Y., 2003). GUSA CVT commercial products are currently available (Ruan, Z. T., 1999) and Zero-Max CVT (Zero-Max, Inc., 2020). Similar designs are detailed in other studies (Fitz, F. A. \& Pires, P. B., 1991; Pires, P. B., 2001) and Benitez (Benitez, F. G., Gutierrez. J., Campillo, G., \& Madronal, P., 2002; Benitez, F. G., Madrigal, J. M., \& Del Castillo, J. M., 2004), which use a number of levers, gear trains and a slotted plate with varying eccentricity. A cam-type ratcheting CVT uses a rotational input from a cam, converts it to an oscillating motion of varying amplitude in the followers and rectifies this motion to a rotational output using a number of one-way clutches.

Lahr (Lahr, D. F., \& Hong, D. W., 2009; Lahr., D., 2013) proposed a cam-based IVT with specially designed cam profiles that ensure a uniform output. Olyaei (Olyaei., A., 2019; Olyaei. A., 2017) proposed a design that is similar to a cam based CVT but which uses a free shaft to transform a rotational input into an oscillatory motion in the followers. Another type of ratcheting CVT uses a scotch yoke mechanism to convert rotation to linear oscillation (Wang, X. F., \& Zhu, W. D., 2014; Wang, X. F., \& Zhu, W. D., 2016). This transmission converts the rotational speed of the input crank to a translational speed for a yoke in a scotch yoke system.

However, most ratcheting CVT are individual designs so there is no systematic design study for these such mechanisms. This paper proposes a variety of innovative coaxial ratcheting CVT design concepts using a systematic method. The designs use linkage, gear and cam drives. 


\section{Design methodology of ratcheting CVT}

This study uses a creative design methodology (Yan, H.-S., 1992; Yan, H.-S., 1998) to produce new designs of ratcheting drive unit for a coaxial ratcheting CVT. A configuration involving a three-bar to five-bar mechanisms with one degree of freedom is proposed.

Using the topological characteristics of existing ratcheting CVT's, the design requirements and design constraints for a ratcheting CVT are determined and the number of links and joints and the types of joints are derived using the equation for degrees of freedom. Number synthesis is used to derive link assortments and the possible variations of available generalized chains are determined. Specific types of members and joints in the atlas of generalized chains are then assigned, subject to specific design requirements and design constraints, to derive the atlas of available specialized chains. These chains are particularized into its corresponding mechanical device and demonstrated in a skeleton drawing. Figure 1 shows the design process for a coaxial ratcheting CVT.

\subsection{Topological characteristics of a ratcheting CVT}

In terms of existing designs with the required specifications, the topological characteristics of ratcheting CVTs are:

(1) The devices are planar mechanism with one degree of freedom and consist of three-bar, four-bar, five-bar and six-bar.

(2) These mechanisms are fixed link $\left(\mathrm{K}_{\mathrm{F}}\right)$, driving link $\left(\mathrm{K}_{\mathrm{I}}\right)$, control link $\left(\mathrm{K}_{\mathrm{C}}\right)$, driven link $\left(\mathrm{K}_{\mathrm{O}}\right)$ and other links $\left(\mathrm{K}_{\mathrm{T}}\right)$.

(3) The joints used are revolute pairs $\left(\mathrm{J}_{\mathrm{R}}\right)$, cam pairs $\left(\mathrm{J}_{\mathrm{A}}\right.$, and sliding pairs $\left(\mathrm{J}_{\mathrm{P}}\right)$.

\subsection{Design requirements and design constraints}

The specifications for a ratcheting CVT are used to produce planar mechanisms with one degree of freedom with threebar, four-bar, and five-bar. Only revolute pairs and cam pairs are used. The design requirements and design constraints for the design process are:

(1) There must be a ground link $\left(\mathrm{K}_{\mathrm{F}}\right)$ as the frame.

(2) There must be a binary link adjacent to the ground link as the input link $\left(\mathrm{K}_{\mathrm{I}}\right)$ and this must be able to rotate through 0 360 degrees.

(3) There must be an output link $\left(\mathrm{K}_{\mathrm{O}}\right)$ adjacent to the ground link.

(4) if a control link $\left(\mathrm{K}_{\mathrm{C}}\right)$ is used, it must be adjacent to the ground link.

(5) The cam pairs for the mechanism must be adjacent to the joints of the ground link or input link.

(6) If there are cam pairs in the mechanism, the maximum number is three.

(7) If there is a control link in the mechanism, the ground link must be at least a ternary link.

(8) If all joints are revolute pairs, the input and output links cannot be in the same four-bar circuit, or the control link will not work.

\subsection{Generalized chains}

According to the Grubler-Kutzbacha criteria, the formula for degrees of freedom for a planar mechanism $\left(F_{p}\right)$ is:

$$
F_{p}=3\left(N_{L}-1\right)-\Sigma N_{J i} C_{p i}
$$

where $N_{L}$ is the number of links, $N_{J i}$ is the number of the $i$-type joints and $C_{p i}$ is the number of degrees of constraint for $i$-type joints.

For a three-bar mechanism, using Equation (1) and the design constraint (6), if two revolute pairs and one cam pair or one revolute pair and three cam pairs are used, the mechanism has one degree of freedom. The numbers and types of joints for four-bar and five-bar mechanisms are listed in Table 1.

The link assortment, $A_{L}$, of a generalized chain is a set of numbers that comprises the number of binary links $\left(L_{L 2}\right)$, ternary links $\left(L_{L 3}\right)$ and quaternary links $\left(L_{L 4}\right)$ in the chain and is expressed as $A_{L}=\left[N_{L 2} / N_{L 3} / N_{L 4} / \ldots\right]$. Different generalized chains with $N_{L}$ links and $N_{J}$ joints, $\left(N_{L}, N_{J}\right)$, are defined by solving Equation (2).

$$
\begin{aligned}
& N_{L 2}+N_{L 3}+\ldots+N_{L i}+\ldots+N_{L m}=N_{L} \\
& 2 N_{L 2}+3 N_{L 3}+\ldots+i N_{L i}+\ldots+m N_{L m}=2 N_{J}
\end{aligned}
$$

where $N_{L i}$ is the number of links with $i$ incident joints, $m$ is the maximum number of joints that are incident to a link and $N_{J}$ is the number of joints. The maximum value of $m$ is expressed as Equation (3).

$$
m_{\max }= \begin{cases}N_{J}-N_{L}+2 & \text { for } \quad N_{L} \leq N_{J} \leq 2 N_{L}-3 \\ N_{L}-1 & \text { for } 2 N_{L}-3 \leq N_{J} \leq N_{L}\left(N_{L}-1\right) / 2\end{cases}
$$

When $m_{\max }$ is derived from Equation (3), all possible link assortments for generalized chains are obtained using Equation (2). 
For a three-bar mechanism, for a $(3,3)$ generalized chain, $N_{L}=3$ and $N_{J}=3$, and according to Equation (3), $m_{\max }$ is equal to 2. Equation (2) gives $N_{L 2}=3$, so this chain has three binary links, $A_{L}=[3]$. For a $(3,4)$ generalized chain, $N_{L}=3$ and $N_{J}=4$, according to Equation (3), and $m_{\max }$ is equal to 3. Equation (2) gives $N_{L 2}=1$ and $N_{L 3}=2$, so the corresponding link assortment is: $A_{L}=$ [1/2], which requires one binary link and two ternary links. Table 1 lists the link assortments for the generalized chains for this study and Figure 2 shows the atlas of $(3,3)$ and $(3,4)$ feasible generalized chains.

\subsection{Specialized chains}

A specialized chain is obtained by assigning specific types of members and joints, subject to the design requirements and design constraints for the available atlas of generalized chains. For $(3,3)$ and $(3,4)$ generalized chains, the design requirements and design constraints are (1), (2), (3), and (5), as mentioned previously. For $(3,3)$ and $(3,4)$ generalized chains, subject to design requirements and design constraints first the chain shown in Figure 2, the ground link $\left(\mathrm{K}_{\mathrm{F}}\right)$ is a frame and the remaining two links are the input link $\left(\mathrm{K}_{\mathrm{I}}\right)$ and the output link $\left(\mathrm{K}_{\mathrm{O}}\right)$. The specialized chain can be obtained is shown in Figure 3.

\subsection{Particularization}

When a feasible specialized chain is determined, each specialized chain is then particularized and the corresponding mechanical devices are defined in a skeleton drawing. For $(3,3)$ and $(3,4)$ specialized chains, there is one new design for a ratcheting drive unit for a ratcheting CVT, as shown in Figure 4.

\subsection{New designs of coaxial ratcheting CVT}

In this section, the output drive unit and the speed control unit are combined in the new designs for a ratcheting drive unit to produce a novel coaxial ratcheting CVT. For the output drive unit for a $(3,3)$ ratcheting drive unit, this study uses an ordinary gear set with a one-way clutch, as shown in Figure 5(a). A planetary gear set with a one-way clutch is used for the $(3,4)$ ratcheting drive unit, as shown in Figure 5(b). The speed control unit does not have a fixed form and accommodates the new designs of ratcheting drive unit that are obtained.

In Figure 5(a), the output link of the ratcheting drive unit is connected to the ordinary gear set and the output link and frame form a joint with a revolute pair. The schematic diagram for a coaxial ratcheting CVT for the new $(3,3)$ design is shown in Figure 6. The main component is an eccentric conical cam $\left(\mathrm{K}_{\mathrm{I}}\right)$. Around the cam are three sets of followers (output links, $\mathrm{K}_{\mathrm{O}}$ ) that reduce the amplitude with which the output speed pulses. An ordinary gear set is used as the output drive unit. An adjuster is used to shift the position of the cam followers in relation to the eccentric conical cam. This affects the swing amplitude of the follower and the transmission output. If a rotational input is applied to the eccentric cam, every output link oscillates using a return spring. Each of the pinions, which are attached to an output link, then rotate back and forth. A one-way clutch is mounted on the pinion to ensure that the output oscillating motion is only transmitted to the output shaft in one direction through the pinion and output gear, as shown in Figure 5(a). The amplitude of the fluctuation in the output link is proportional to the action radius of the eccentric cam and this is adjusted by moving the adjuster position. The greater the amplitude of the fluctuation when the output link moves closer to the output side, the smaller is the amplitude of the fluctuation when the output link moves closer to the input side. The output speed is changed by moving the adjuster along the axial direction of the eccentric cam.

In Figure 5(b), the output link of the ratcheting drive unit is connected to the carrier of the planetary gear train and the output link and frame are joined using a cam pair. The schematic diagram of a new design of coaxial ratcheting CVT for $(3,4)$ is shown in Figure 7. The core of this transmission is an eccentric positive return cam $\left(\mathrm{K}_{\mathrm{I}}\right)$, , around which are three sets of followers (output link $\mathrm{K}_{\mathrm{O}}$ ). One end of the output link is constrained to maintain contact with the eccentric cam profile and the other end is located in the groove of the frame $\left(\mathrm{K}_{\mathrm{F}}\right)$. A roller that is mounted on the pinion of the speed adjuster is installed in a groove on the output link to act as a fulcrum for the output link. When a rotational input is applied to the eccentric cam, every output link swings with respect to the output link fulcrum. Each of the planetary gears, which are attached to an output link, then rotate back and forth. A one-way clutch is mounted on the planet gear to ensure that the output oscillating motion is only transmitted to the output shaft in one direction through the plenary gear set, as shown in Figure 5(b). The amplitude of fluctuation in the output link is proportional to the position of the output link fulcrum so the amplitude is increased by turning the speed adjust gear to change the position of the output link fulcrum. The amplitude of the fluctuation increases when the output link fulcrum moves closer to the input shaft. For a four-bar and five-bar mechanism, according the results of the proposed design process are shown in Table 2 to Table 5 .

\section{Kinematic analysis}

This section details a kinematic analysis of the $(3,4)$ coaxial ratcheting CVT using a vector loop approach (Hall, A. S. Jr., 1987), in order to verify its feasibility. The vector loop approach firstly defines the vector position equation for a 
mechanism onto a fixed coordinate system and divides a vector position equation into two scalar equations. For a specific position for the input link, the scalar equations are solved to derive the position of the remaining links.

The first and second derivatives of the scalar position equations define the velocity and acceleration equations for the mechanism. For a specific velocity and acceleration for the input link, these equations are solved to determine the velocity and acceleration of the remaining links.

Figure 8 shows an $x$ - $y$ coordinate system with origin $O$ at the center of the input shaft. The angle for each vector is measured counter-clockwise with respect to the positive $x$-axis. All vectors are defined as follows.

$\vec{r}_{1}$, from the fulcrum of the output link to the center of input shaft

$\vec{r}_{2}$, from the center of the input shaft to the center of eccentric cam

$\vec{r}_{3}$, from the center of the eccentric cam to the center of the cam groove

$\vec{r}_{4}$, from the lower end of the output link to the fulcrum of output link

$\vec{r}_{5}$, attached to the output link

$\vec{r}_{6}$, from the center of the planet gear to the center of input shaft

The vector loop position equations are:

$$
\begin{aligned}
& \vec{r}_{1}+\vec{r}_{2}+\vec{r}_{3}+\vec{r}_{4}=0 \\
& \vec{r}_{2}+\vec{r}_{3}+\vec{r}_{5}+\vec{r}_{6}=0
\end{aligned}
$$

Grashof's theorem states that a four-bar mechanism has at least one revolving link if the sum of the length of its shortest and longest links is less than or equal to the sum of the length of the other two links. Grashof's criterion is: $s+l \leq p+q$, where $s$ is length of the shortest bar, $l$ is length of the longest bar and $p$ and $q$ are lengths of the intermediate bars. In Figure 8, $\vec{r}_{1}, \vec{r}_{2}, \vec{r}_{3}$, and $\vec{r}_{4}$ form a 4-bar loop. If the eccentricity of the eccentric cam, $r_{2}$, is for the shortest bar as the crank, it is equivalent to a crank-rocker mechanism.

To separate each of Equations (4) into scalar component equations, the loop equations are:

$$
\begin{aligned}
& r_{1} \cos \theta_{1}+r_{2} \cos \theta_{2}+r_{3} \cos \theta_{3}+r_{4} \cos \theta_{4}=0 \\
& r_{1} \sin \theta_{1}+r_{2} \sin \theta_{2}+r_{3} \sin \theta_{3}+r_{4} \sin \theta_{4}=0 \\
& r_{2} \cos \theta_{2}+r_{3} \cos \theta_{3}+r_{5} \cos \theta_{5}+r_{6} \cos \theta_{6}=0 \\
& r_{2} \sin \theta_{2}+r_{3} \sin \theta_{3}+r_{5} \sin \theta_{5}+r_{6} \sin \theta_{6}=0
\end{aligned}
$$

where $\theta_{1}, \theta_{2}, \theta_{3}, \theta_{4}, \theta_{5}$, and $\theta_{6}$ define the angle of the each corresponding link with respect to the positive x-axis. The constants in Equation (5) are $\theta_{1}, r_{1}, r_{2}, r_{3}, r_{5}$, and $r_{6}$, and the variables are $\theta_{2}, \theta_{3}, \theta_{4}=\theta_{5}, \theta_{6}$, and $r_{4}$, where $\theta_{2}$ is the input angle. Differentiating Equation (5) with respect to time gives the velocity equations:

$$
\begin{aligned}
& -r_{2} \sin \theta_{2} \dot{\theta}_{2}-r_{3} \sin \theta_{3} \dot{\theta}_{3}+\dot{r}_{4} \cos \theta_{4}-r_{4} \sin \theta_{4} \dot{\theta}_{4}=0 \\
& r_{2} \cos \theta_{2} \dot{\theta}_{2}+r_{3} \cos \theta_{3} \dot{\theta}_{3}+\dot{r}_{4} \sin \theta_{4}+r_{4} \cos \theta_{4} \dot{\theta}_{4}=0 \\
& -r_{2} \sin \theta_{2} \dot{\theta}_{2}-r_{3} \sin \theta_{3} \dot{\theta}_{3}-r_{5} \sin \theta_{5} \dot{\theta}_{5}-r_{6} \sin \theta_{6} \dot{\theta}_{6}=0 \\
& r_{2} \cos \theta_{2} \dot{\theta}_{2}+r_{3} \cos \theta_{3} \dot{\theta}_{3}+r_{5} \cos \theta_{5} \dot{\theta}_{5}+r_{6} \cos \theta_{6} \dot{\theta}_{6}=0
\end{aligned}
$$

where $\dot{\theta}_{2}, \dot{\theta}_{3}, \dot{\theta}_{4}, \dot{\theta}_{5}$, and $\dot{\theta}_{6}$ are the angular velocity of each corresponding link and $\dot{r}_{4}$ is the sliding speed of the output link relative to the fulcrum. When the position analysis is completed, the known values in Equation (6) are $r_{2}$, $r_{3}, r_{4}, r_{5}, r_{6}, \theta_{2}, \theta_{3}, \theta_{4}=\theta_{5}$, and $\theta_{6}$ and the unknown variables are $\dot{\theta}_{2}, \dot{\theta}_{3}, \dot{\theta}_{4}=\dot{\theta}_{5}, \dot{\theta}_{6}$, and $\dot{r}_{4}$, where $\dot{\theta}_{2}$ is the angular velocity of the input.

The output drive unit for the proposed design is shown in Figure 5(b). When the number of teeth of each gear is specified the gear ratio of the planetary gear set can be calculated. The output speed, $\omega_{\text {out }}$, of the coaxial ratcheting CVT is written as:

$$
\omega_{\text {out }}=\left(1-N_{g 2} N_{g 4} / N_{g 3} N_{g 5}\right) \dot{\theta}_{6}
$$


where $N_{g 2}, N_{g 3}, N_{g 4}$, and $N_{g 5}$ are the number of teeth on the fixed sun gear 2, planetary gears 3 and 4 and the output gear 5. As shown in Figure 9, this design example has value of considered as: $r_{2}=7.5 \mathrm{~mm}, r_{3}=20 \mathrm{~mm}, r_{5}=70 \mathrm{~mm}$, $r_{6}=75 \mathrm{~mm}, N_{g 2}=100, N_{g 3}=20, N_{g 4}=16$, and $N_{g 5}=104$.

The vector loop approach is a systematic process that is suited to a computer program. The Newton-Raphson method is used to solve all unknowns for this design example. The input speed is $\dot{\theta}_{2}=1000 \mathrm{rpm}$. Figure 9 shows the simulation results for the example. The three output links are located around the cam profile with a phase difference of 120 degrees so there are three phases of oscillating output. The curves for the three different amplitudes in Figure 9 are used to adjust the fulcrum position for the output link, so $r_{1}=60 \mathrm{~mm}, r_{1}=50 \mathrm{~mm}$ and $r_{1}=40 \mathrm{~mm}$. When the position of fulcrum of the output link is close to the input shaft, the swing amplitude of the output link and the output speed are increased. If the fulcrum for the output link is moved away from the input shaft, swing amplitude of the output link and the output speed are decreased. The output speed is continuously changed by adjusting the position of fulcrum for the output link.

\section{Conclusion}

This study proposes a variety of innovative coaxial ratcheting CVT design concepts using a creative design methodology. This study focuses on three-bar to five-bar mechanisms and a total of 10 new designs of ratcheting drive unit for a coaxial ratcheting CVT are derived. A linkage, gear and cam mechanism is used for these new designs. An output drive unit and a speed control unit are used to produce a new design for a coaxial ratcheting CVT.

A design example is used to verify the feasibility of the system. The design process for this study can be used to design a ratcheting CVT and the results show that the options for this type of mechanism are many.

\section{References}

Benitez, F. G., Gutierrez. J., Campillo, G., \& Madronal, P. (2002). Variable Continuous Transmission System. US Patent 6,371,881 B1.

Benitez, F. G., Madrigal, J. M., \& Del Castillo, J. M. (2004). Infinitely Variable Transmission of Ratcheting Drive Type Based on One-Way Clutches. ASME Journal of Mechanical Design, Vol. 126, 673-682.

Fitz, F. A., \& Pires, P. B. (1991). A geared Infinitely Variable Transmission for Automotive Applications. SAE paper No. 910407, 1-7.

Hall, A. S. Jr. (1987). Notes on Mechanism Analysis. Waveland Press, ISBN 0-88133-270-4.

Lahr, D.F. \& Hong, D.W. (2009). Operation and Kinematic Analysis of a Cam-Based Infinitely Variable Transmission. ASME Journal of Mechanical Design, Vol. 131, 081009.

Lahr., D. (2013). Cam-Based Infinitely Variable Transmission. US Patent 8.425,364 B2.

Matsumoto, S., Inoue, N., \& Tsukada, Y. (2003). Continuously Variable Transmission for Bicycles. United States Patent Application 20030221892.

Olyaei. A. (2017). Continuously Variable Transmission. US Patent 9816593 B2.

Olyaei., A. (2019). Novel continuously Variable Transmission Mechanism. SN Applied Sciences, 1:1032. Retrieved from https://doi.org/10.1007/s42452-019-1081-4

Pires, P.B. (2001). Transmission Ratio Changing Apparatus and Method. US Patent 4,983,151.

Ruan, Z.T. (1999). Design and Selection Guide of Mechanical Continuously Variable Transmission. Chemical Industry Press. Beijing.

Wang, X. F., \& Zhu, W. D. (2014). Design, Modeling, and Simulation of a Geared Infinitely Variable Transmission. ASME Journal of Mechanical Design, 136 (7), 071011.

Wang, X. F., \& Zhu, W. D. (2016). Design, Modeling, and Experimental Validation of a Novel Infinitely Variable Transmission Based on Scotch Yoke Systems. Transactions of the ASME, Vol. 138/015001, 1-8.

Yan, H.-S. (1992). A methodology for Creative Mechanical Design. Mechanism and Machine Theory. Vol. 27, No. 3, 235-242.

Yan, H.-S. (1998). Creative Design of Mechanical Devices. Springer-Verlag, ISBN 981-3083-57-3.

Yates, W.A. (2008). Design Principles of a Four-bar Crank Continuously Variable Transmission. ASME 2008 2nd International Conference on Energy Sustainability, Jacksonville, Florida, August. Vol. 1, 419-438.

Zero-Max motion control products. (2020, February). Retrieved from https://www.zero-max.com/cd-adjustable-speeddrives\#1 


\section{Appendix}

The results of the four-bar and five-bar new designs are shown in appendix Table 2 to Table 5.

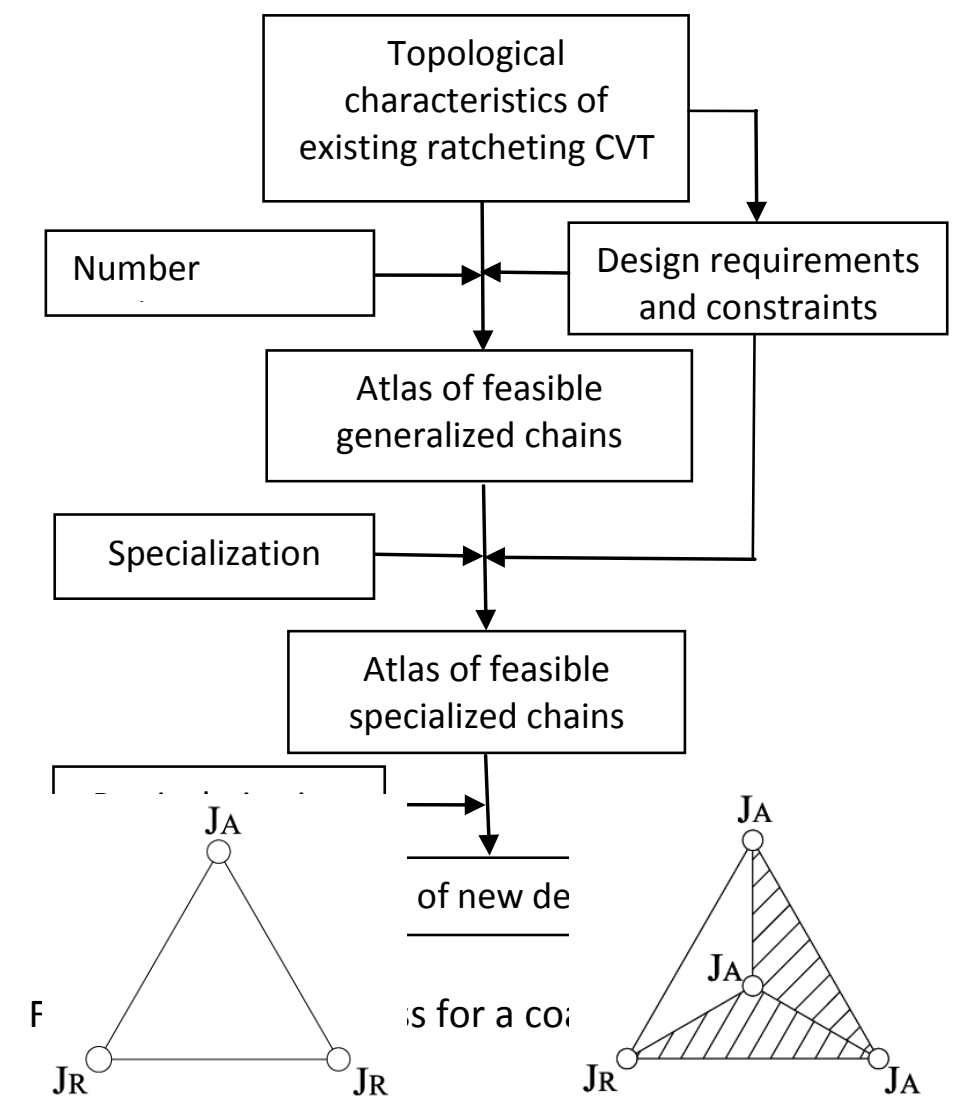

(a) $(3,3)$ generalized chain

(b) (3.4) generalized chain

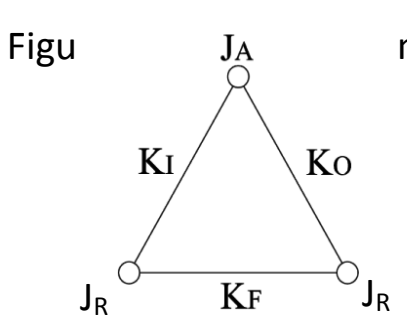

nd $(3,4)$ feasi

(b) (3 2) cnarializad rhain

Figur
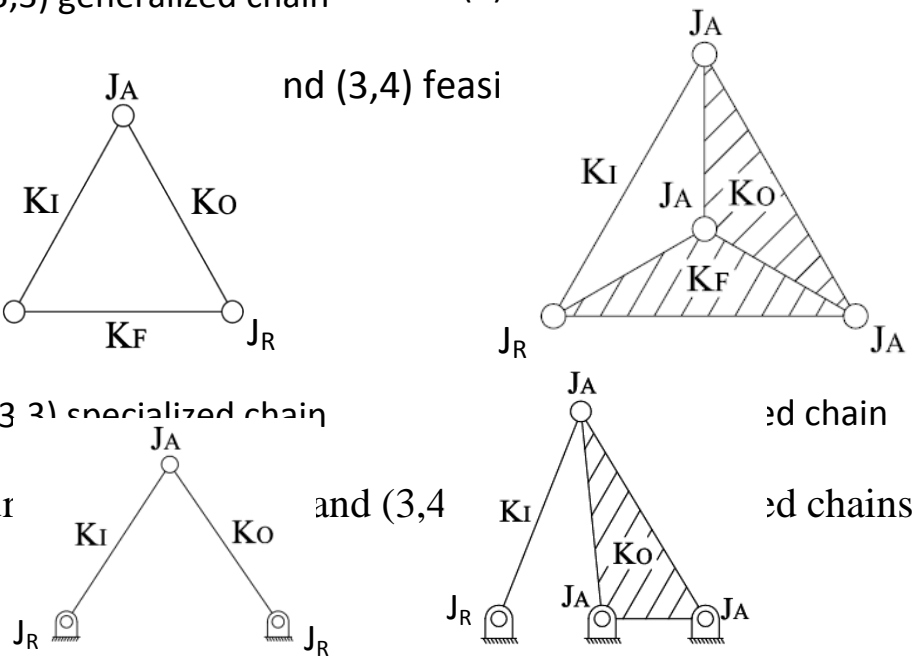

(b) $(3,3)$ new designs

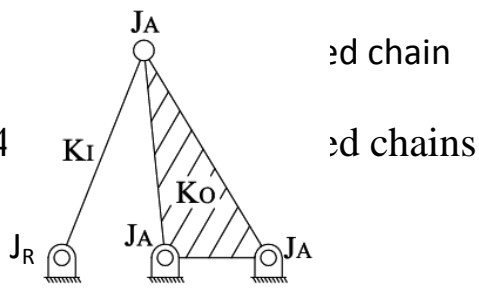

(a) $(3,4)$ new designs

Figure 4 New designs for a $(3,3)$ and $(3,4)$ ratcheting drive 


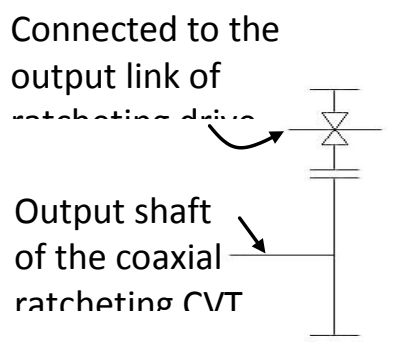

(a)

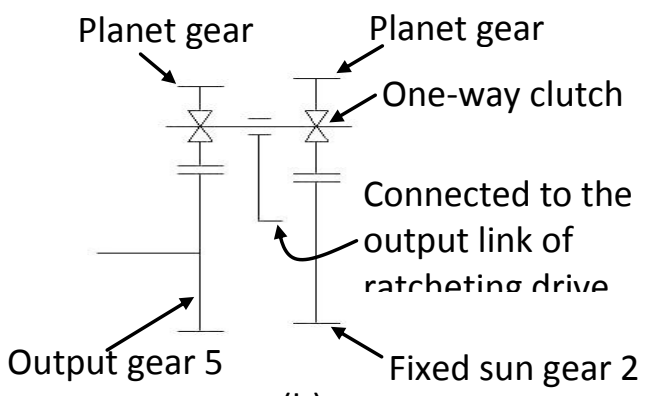

(b)

Figure 5 Output drive units for $(3,3)$ and $(3,4)$ ratche drive units
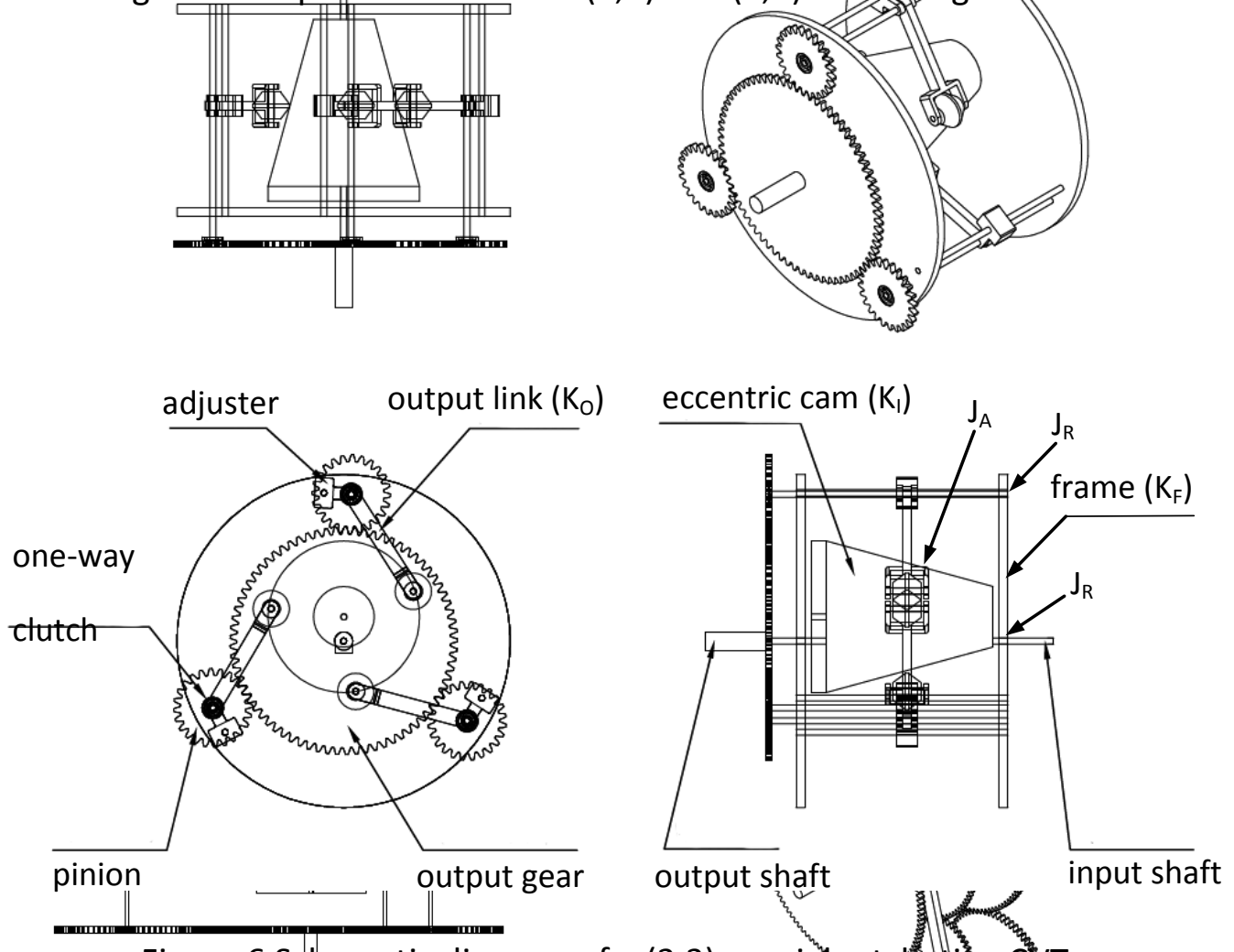

Figure 6 Schematic diagram of a $(3,3)$ coaxialmkatchenti

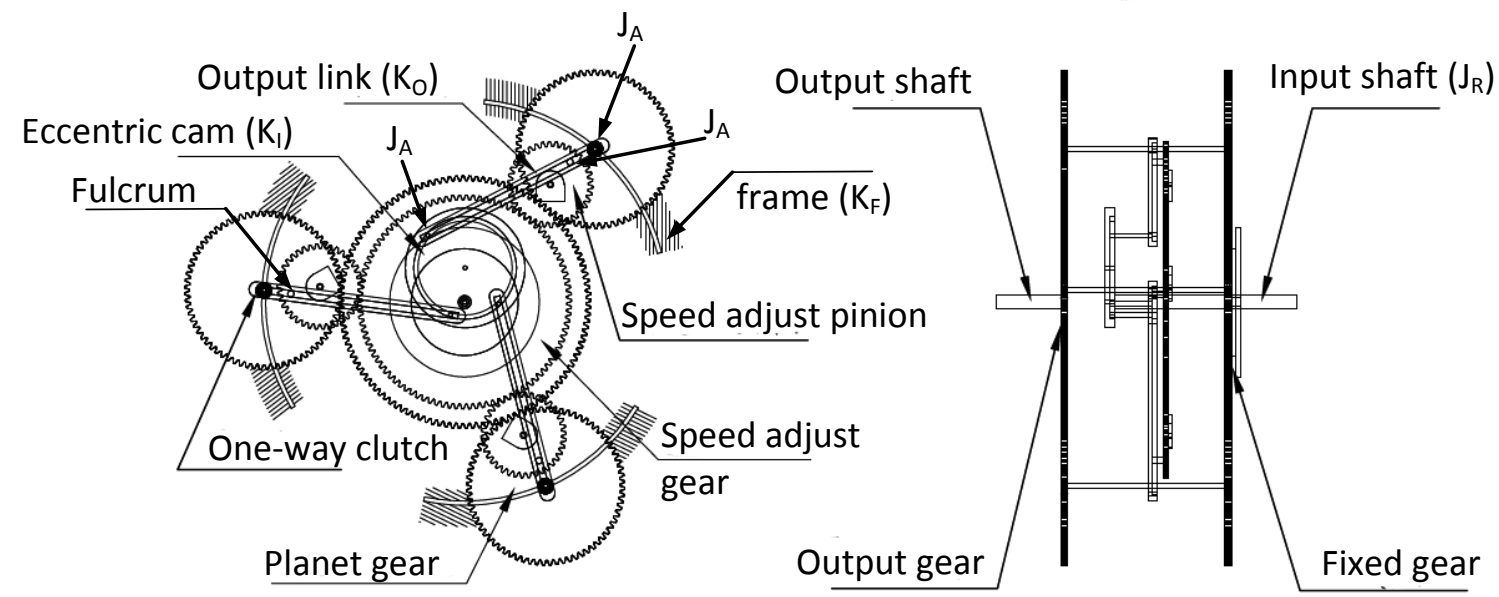

Figure 7 Schematic diagram of a $(3,4)$ coaxial ratcheting CVT 


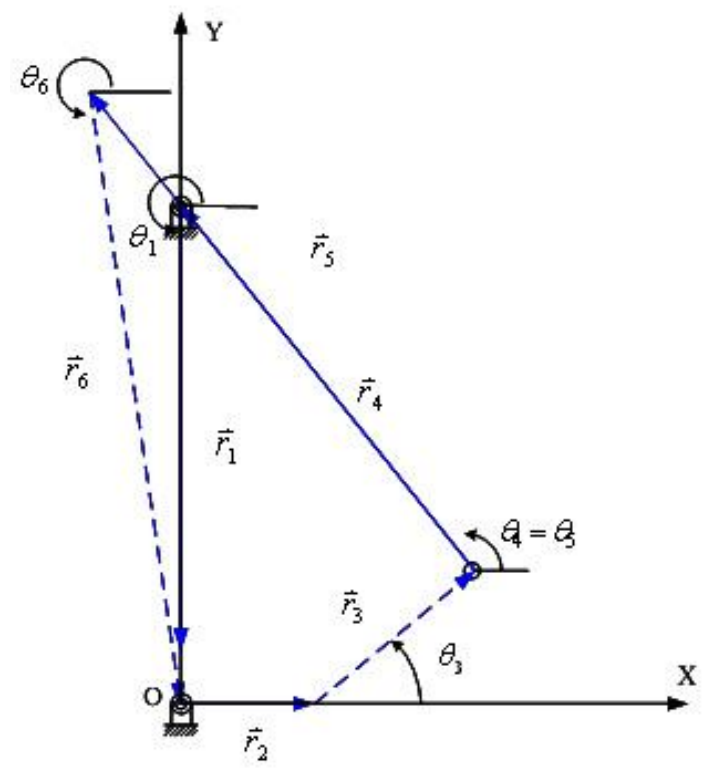

Figure 8 Vector loop of the $(3,4)$ new design

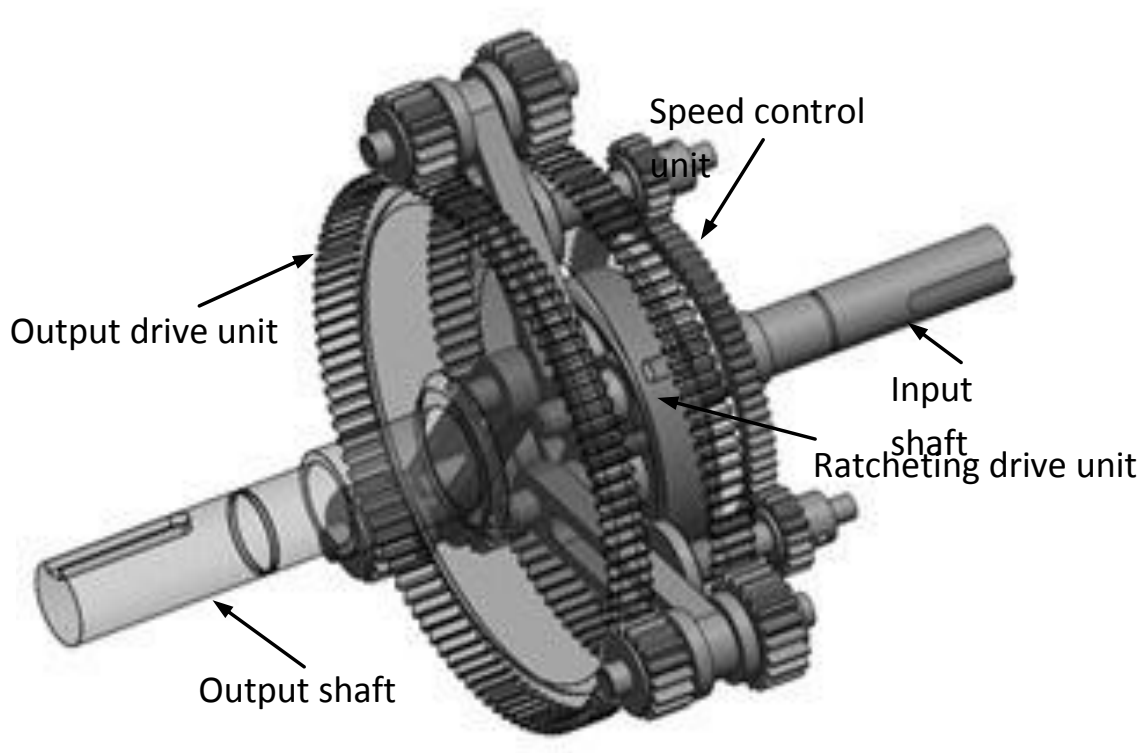

Figure 9 Design example for a $(3,4)$ coaxial ratcheting CVT

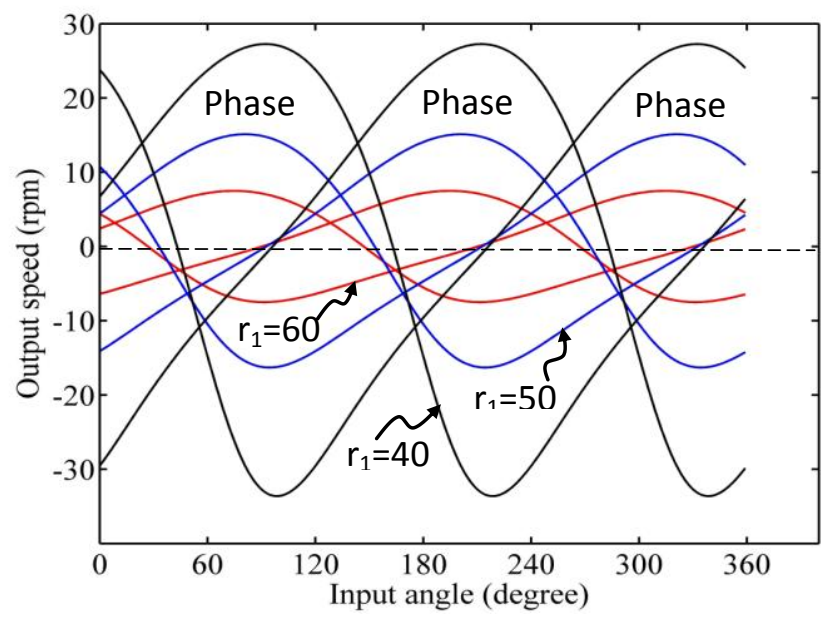

Figure 10 Simulation results for a $(3,4)$ coaxial ratcheting CVT 
Table 1 Link assortments and numbers and types of joints

\begin{tabular}{|l|l|l|l|}
\hline \multirow{2}{*}{$\begin{array}{l}\text { Number } \\
\text { of links }\end{array}$} & $\begin{array}{l}\text { Feasible } \\
\text { generalized } \\
\text { chain }\left(N_{L}, N_{J}\right)\end{array}$ & $\begin{array}{l}\text { Link assortment } \\
\left(A_{L}\right)\end{array}$ & $\begin{array}{l}\text { Number and } \\
\text { type of joints }\end{array}$ \\
\hline \multirow{2}{*}{ Three-bar } & $(3,3)$ & {$[3]$} & $2 \mathrm{~J}_{\mathrm{R}}, 1 \mathrm{~J}_{\mathrm{A}}$ \\
\cline { 2 - 4 } & $(3,4)$ & {$[1 / 2]$} & $1 \mathrm{~J}_{\mathrm{R}}, 3 \mathrm{~J}_{\mathrm{A}}$ \\
\hline \multirow{2}{*}{ Four-bar } & $(4,4)$ & {$[4]$} & $4 \mathrm{~J}_{\mathrm{R}}$ \\
\cline { 2 - 4 } & $(4,5)$ & {$[2 / 2]$} & $3 \mathrm{~J}_{\mathrm{R}}, 2 \mathrm{~J}_{\mathrm{A}}$ \\
\hline \multirow{2}{*}{ Five-bar } & $(5,6)$ & {$[3 / 2]$} & $5 \mathrm{~J}_{\mathrm{R}}, 1 \mathrm{~J}_{\mathrm{A}}$ \\
\cline { 2 - 4 } & $(5,7)$ & {$[2 / 2 / 1],[3 / 0 / 1]$} & $4 \mathrm{~J}_{\mathrm{R}}, 3 \mathrm{~J}_{\mathrm{A}}$ \\
\hline
\end{tabular}

Table 2 New design for a $(4,4)$ coaxial ratcheting CVT

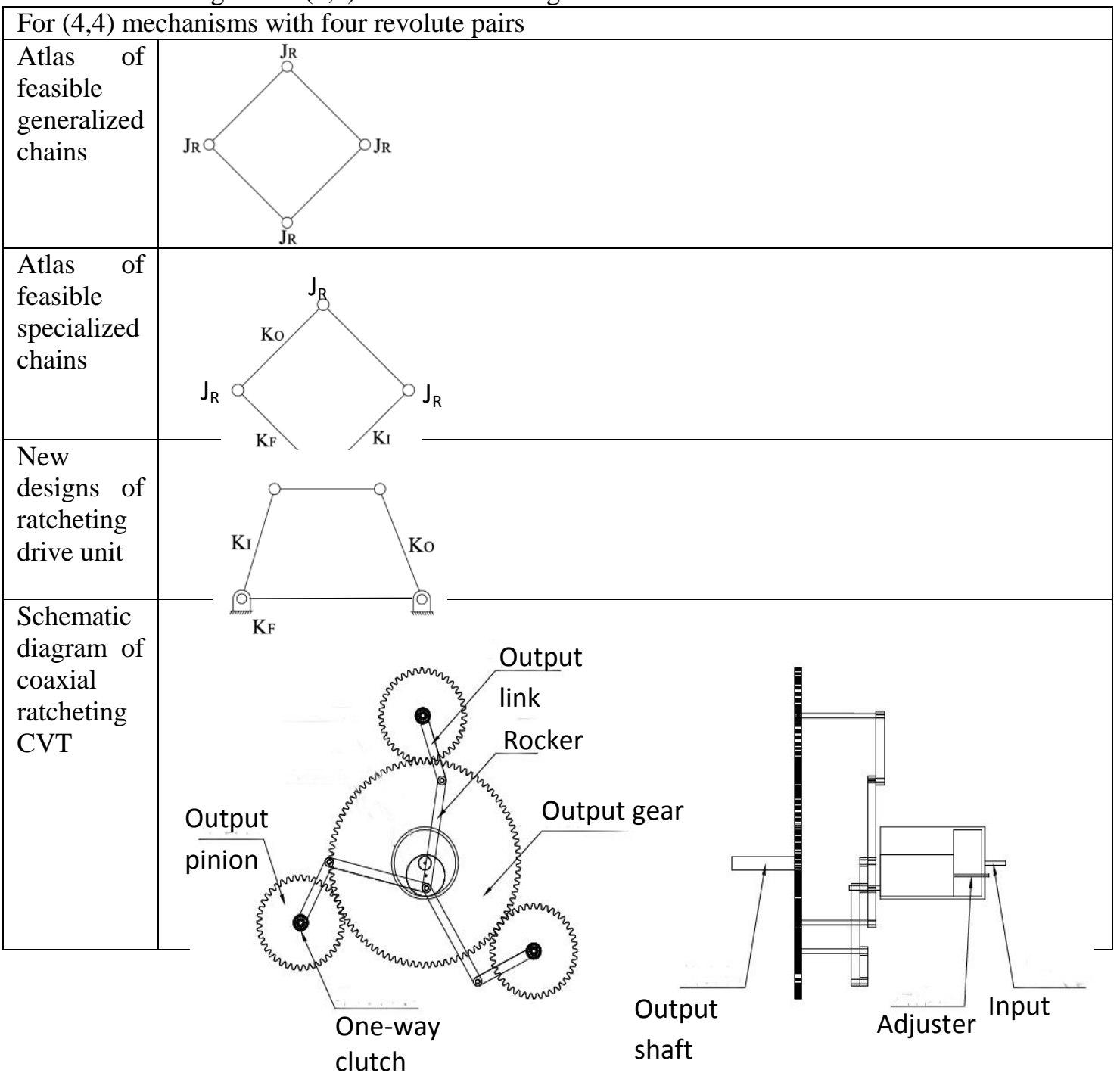


Table 3 New design for a $(4,5)$ coaxial ratcheting CVT

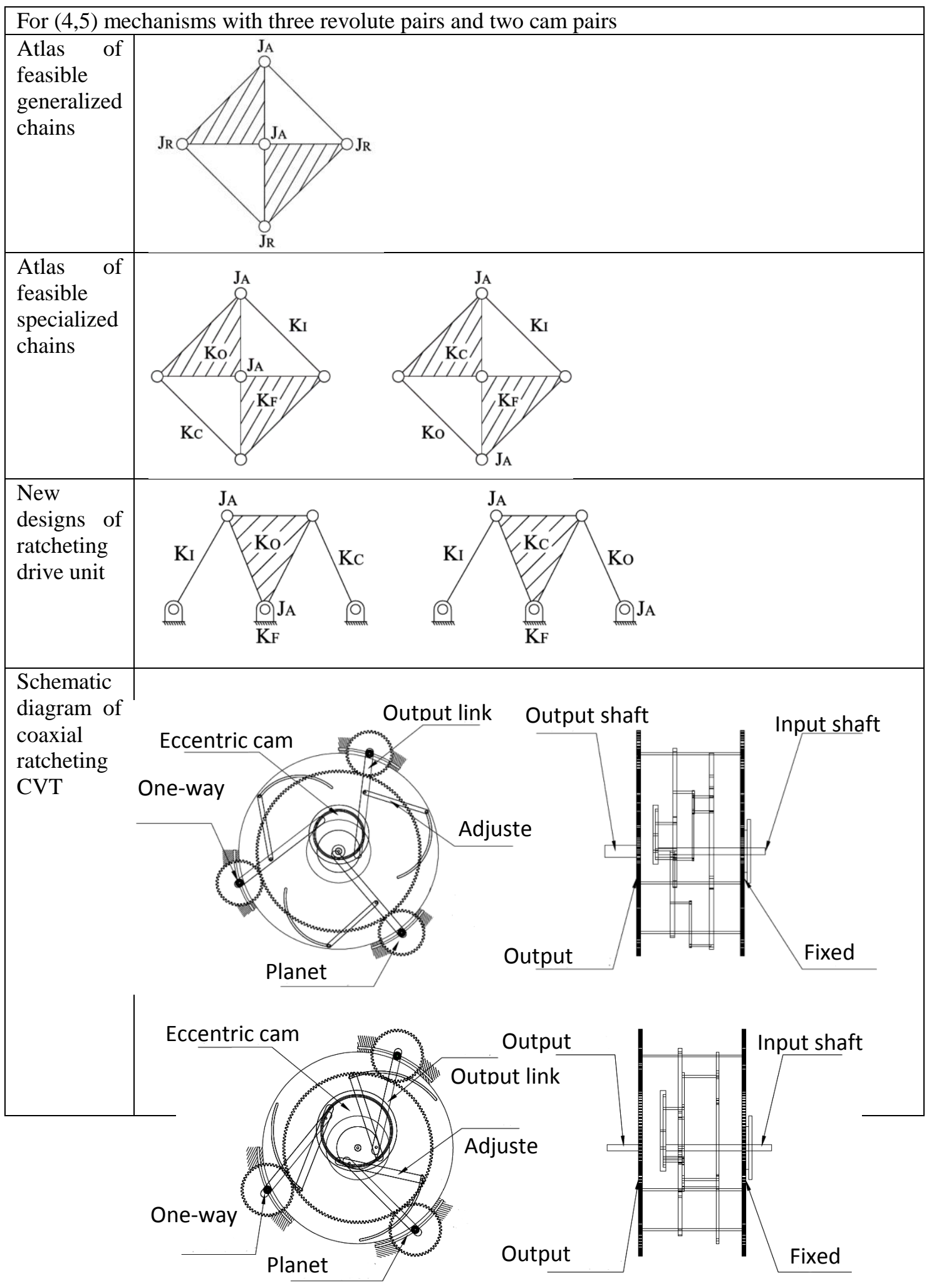


Table 4 New design for a $(5,6)$ coaxial ratcheting CVT

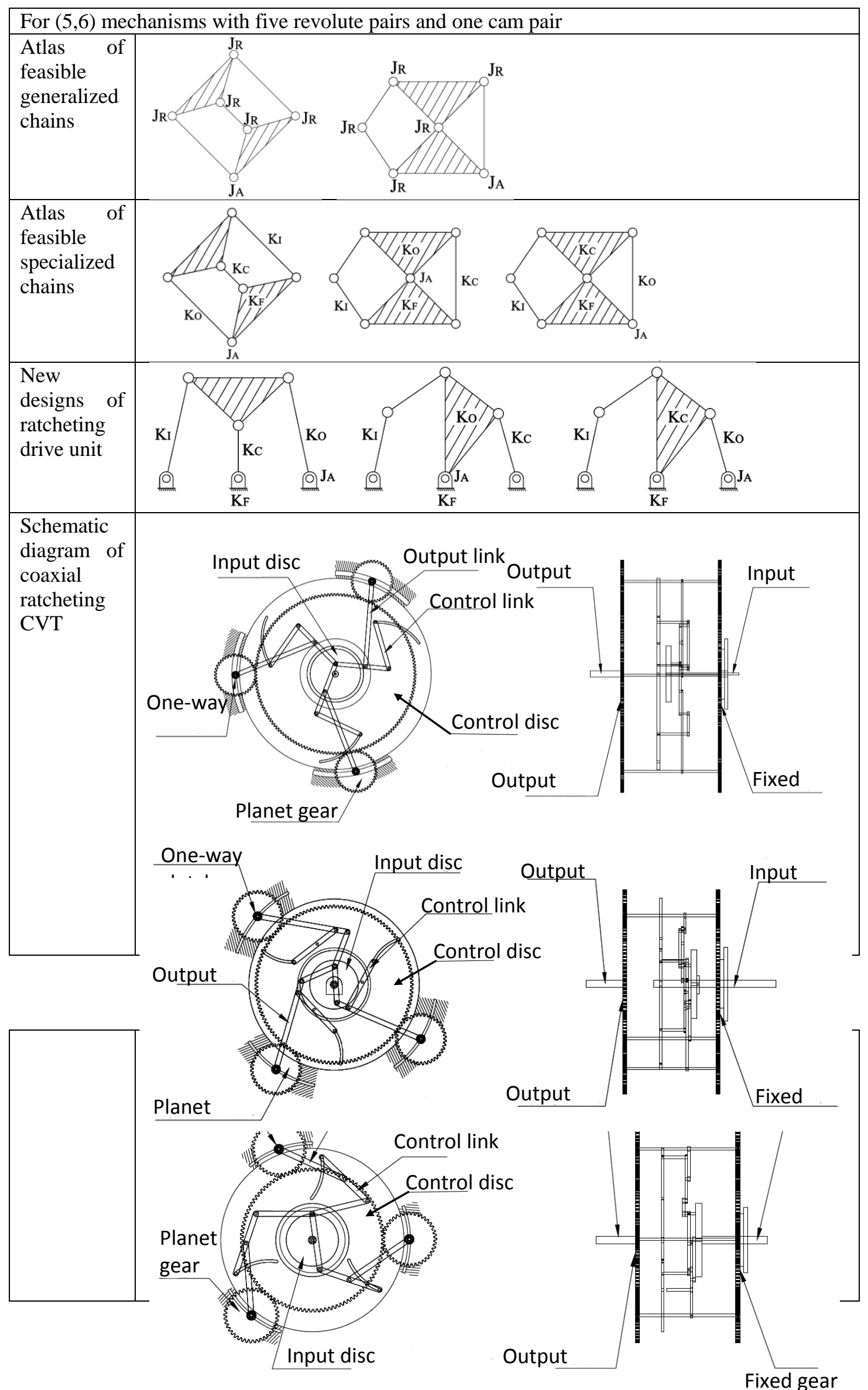


Table 5 New design for a $(5,7)$ coaxial ratcheting CVT

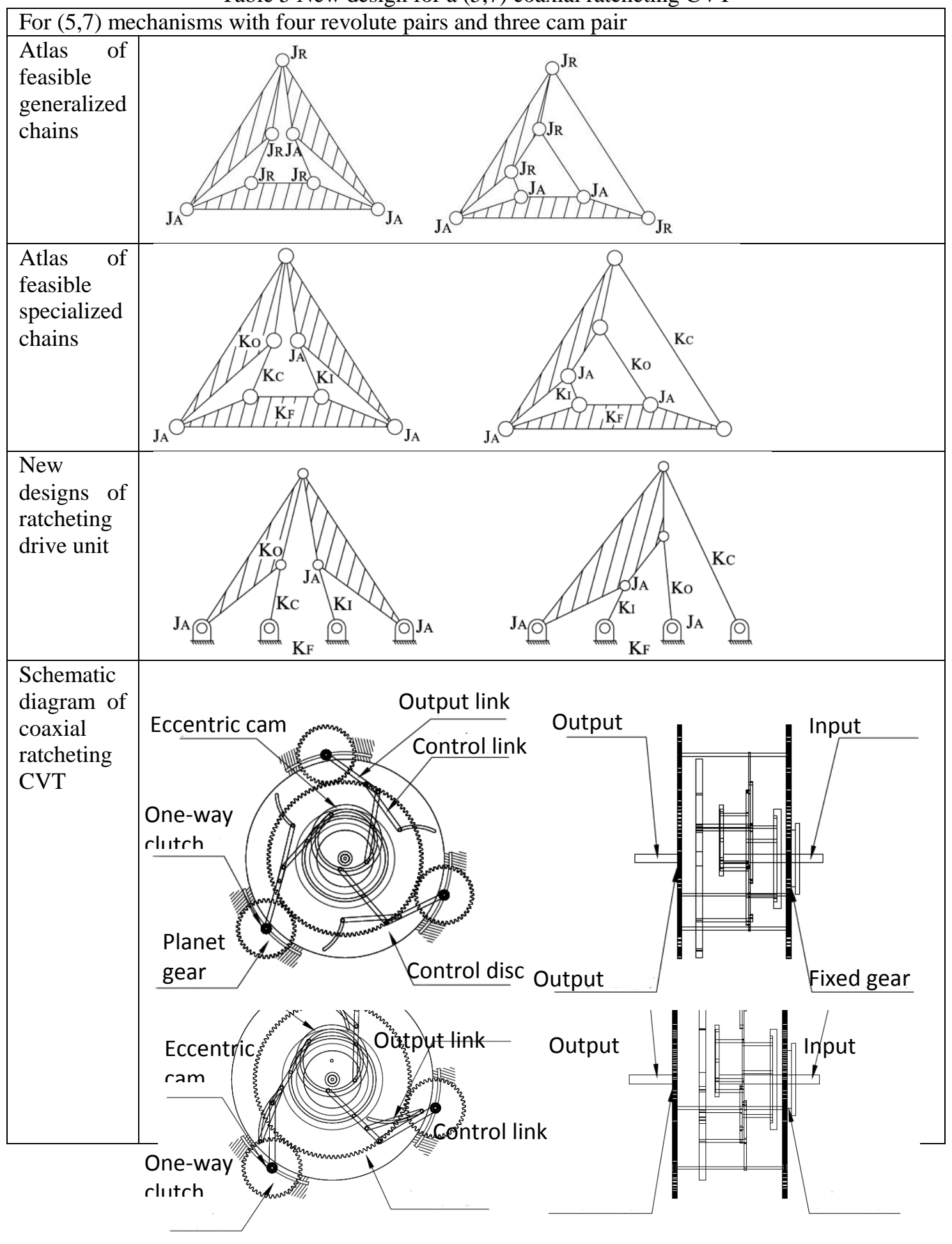

Planet gear

Control disc Output

Fixed gear 